Int. J. Dev. Biol. 54: 1241-1250 (2010)

doi: $10.1387 / \mathrm{ijdb} .093010 \mathrm{mb}$

\title{
Leg regeneration in Drosophila abridges the normal developmental program
}

\author{
MANEL BOSCH ${ }^{1,2,3}$, SARAH-ANNE BISHOP1, JAUME BAGUÑ ${ }^{2}$ and JUAN-PABLO COUSO1,* \\ ${ }^{1}$ School of Life Sciences, University of Sussex, Falmer, Brighton, U.K., \\ ${ }^{2}$ Department de Genètica, Facultat de Biología, Universitat de Barcelona, Barcelona and \\ ${ }^{3}$ Serveis Científic Tècnics, Universitat de Barcelona, Facultat de Biología, Barcelona, Spain
}

\begin{abstract}
Regeneration of lost body parts has traditionally been seen as a redeployment of embryonic development. However, whether regeneration and embryonic development are controlled by identical, similar or different genetic programmes has not been fully tested. Here, we analyse proximal-distal regeneration in Drosophila leg imaginal discs using the expression of positional markers, and by cell-lineage experiments, and we compare it with the pattern already known in normal development. During regeneration, the first proximal-distal positional markers reappear in overlapping patterns. As the regenerate expands, they segregate and further markers appear until the normal pattern is produced, following a proximal to distal sequence that is in fact the reverse of normal leg imaginal disc development. The results of lineage tracing support this interpretation and show that regenerated structures derive from cells near the wound edge. Although leg development and leg regeneration are served by a set of identical genes, the ways their proximal-distal patterns are achieved are distinct from each other. Such differences can result from similar developmental gene interactions acting under different starting conditions.
\end{abstract}

KEY WORDS: regeneration, gene expression, pattern formation, Drosophila, leg disc

\section{Introduction}

Regeneration is one of the most fascinating phenomena in biology. All animals have the ability to develop, most have some ability to repair damaged structures, but only a minority are able to regenerate completely lost organs (reviewed in Sanchez Alvarado, 2000). The lack of experimental systems amenable to genetic analysis for the study of regeneration has hampered its understanding and the answering of crucial questions. It is not known if regeneration proceeds similarly in all animals; that is, if there are universal rules and constraints to regeneration; or what are its relationships to normal developmental processes. Humans show a limited ability to regenerate some structures, and the possibility to uncap hidden regenerative abilities has therapeutic implications.

Drosophila limbs and appendages develop from imaginal discs, transitory larval structures that transform into their corresponding adult structures during metamorphosis. Imaginal discs have a remarkable capacity for regeneration after damage, cutting or partial amputation when cultured inside adult individuals (see methods). Numerous studies in the 1970-80s produced several models and rules that formally explained the main outcomes of disc regeneration, such as the Polar Coordinate Model (PCM; French et al., 1976), with subsequent revisions in Bohn, 1976; Schubiger and Schubiger, 1978; Strub, 1979; Bryant et al., 1981; Couso etal., 1993; and the Boundary Model (BM; Meinhardt, 1982, 1983). In the leg imaginal disc, removal of its distal (central) part, followed by culture in a growth permissive environment, induces the regeneration of the lost (distal) structures from the proximal cut edge (terminal regeneration; French et al., 1976; Schubiger and Schubiger, 1978; Strub, 1979). If the distal part of the leg disc is cultured alone, distal structures will be produced from the cut edge leading to a duplication of the fragment. The fact that disc fragments bearing proximal parts regenerate the distal elements, whereas distal fragments duplicate these elements and do not regenerate the proximal ones, has been termed 'distal transformation', which appears to be the rule in terminal regeneration. An extrapolation of this rule might suggest that the order

Abbreviations used in this paper: AEL, after egg laying; EGFR, epidermal growth factor receptor; PD, proximal-distal.

\footnotetext{
*Address correspondence to: Juan-Pablo Couso. School of Life Sciences, University of Sussex, Falmer, Brighton, BN1 90G. U.K. Tel: +44-1273-877-448. Fax: +44-1273-877586. e-mail: j.p.couso@sussex.ac.uk - web: http://www.sussex.ac.uk/biology/profile114323.html
} 
of appearance of regenerated structures would be, at first, from proximal to distal. However, even if distal cells would not normally switch to proximal fates in terminal regeneration, they have been shown to do so during intercalary regeneration (i.e. regeneration that follows after tissue between a proximal and a distal point is removed and the two stumps joined together) as shown in cockroaches (Bohn, 1976; Truby, 1985), the phasmid Carausius(Bart, 1988) and crickets (Mito et al., 2002).

Insight into the molecular mechanism of leg imaginal disc regeneration had to wait for the molecular understanding of normal leg imaginal disc development. In the developing leg disc, hedge$h o g(h h)$ is expressed in the posterior compartment cells of the disc, and induces decapentaplegic (dpp) and wingless (wg) expression in adjacent dorsal and ventral cells, respectively (Basler and Struhl, 1994). The combined activity of Wg and Dpp signalling establishes, in second instar larval discs, the expression of Distalless (DII) in the centre of the disc, and of dachsund (dac) more proximally (Fig. 1A) (Diaz-Benjumea et al., 1994; Campbell and Tomlinson, 1995; Lecuit and Cohen, 1997). Lineage tracing (Weigman and Cohen,
1999) shows that the 'distal transformation rule' also applies to normal development at this early stage. Proximal-most cells (which do not express $D /$ ) can switch their pattern of gene expression as the disc grows and acquire D//expression, and hence, distal fates, while distal cells do not switch off D// expression, nor convert to proximal-most fates (Wu and Cohen, 1999). Later on, at the early third instar stage, DII, Dpp and Wg activity induce expression of vein $(v n)$, the epidermal growth factor receptor (EGFR) ligand, at the centre of the disc (Campbell, 2002; Galindo et al., 2002). This sets in motion a Wg- and Dpp-independent intercalation process that results in the proximal-distal (PD) subdivision of the D//domain through overlapping ring-like gene expression patterns (Campbell, 2002; Galindo etal., 2002; reviewed in Kojima, 2004). Briefly, tarsal and pretarsal (distalmost) PD fates are determined by a gradient of EGFR activity in a distal to proximal direction as revealed by the activation of the distal markers $C 15$, aristaless (al) and $\operatorname{Bar}(B)$ earlier than more proximal markers such as bric-a-brac (bab), rotund ( $r n$ ) and apterous (ap)(Fig. $1 \mathrm{~B}-\mathrm{C}$ ) (reviewed in Couso and Bishop, 1998; Galindo and Couso, 2000; Kojima, 2004).
A

B
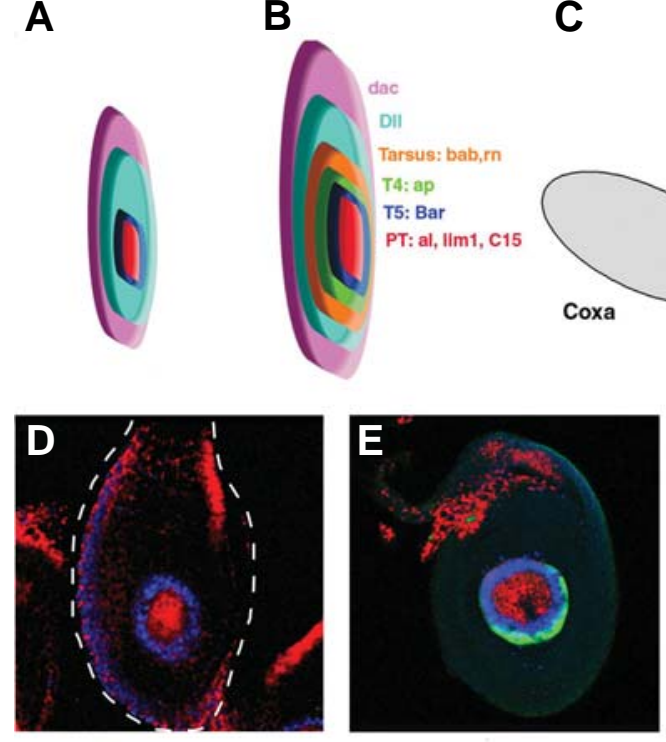

C
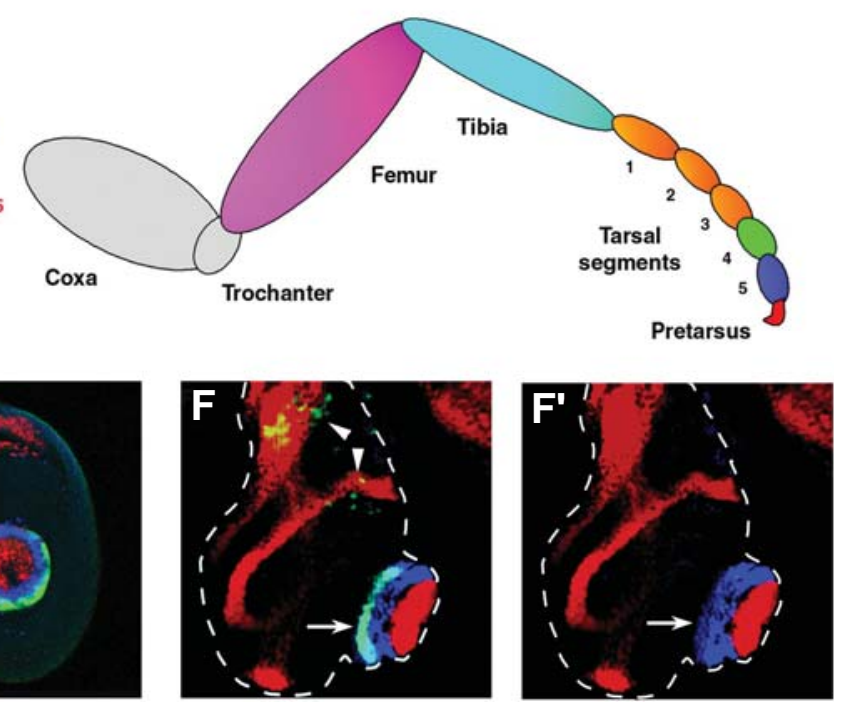

Fig. 1. Wild-type and regenerative development. Diagrams of (A) early and $\mathbf{( B )}$ late third-instar leg imaginal discs. Dorsal is up and distal right. Relevantgene expression domains are colour coded along the leg proximaldistal axis. (C) Adult leg with its segments coloured according to the gene expression domains in (B). (D) Triple staining of an early third-instar leg disc showing al (red) and Bar (blue), and absence of ap (green). The dashed white line indicates the disc contour.

G

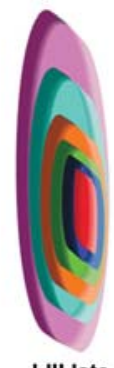

LIII late

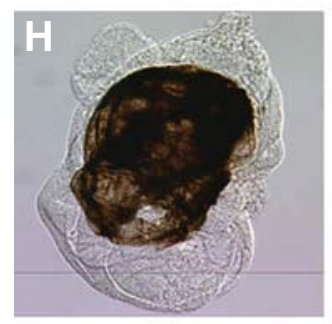

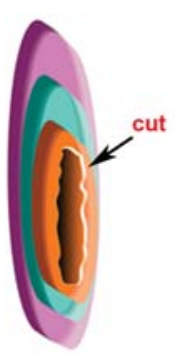

'ap -fragment'

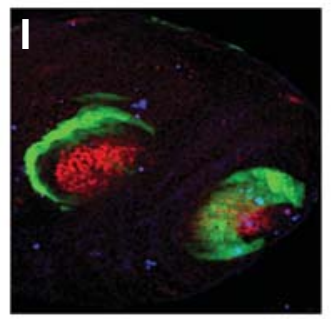

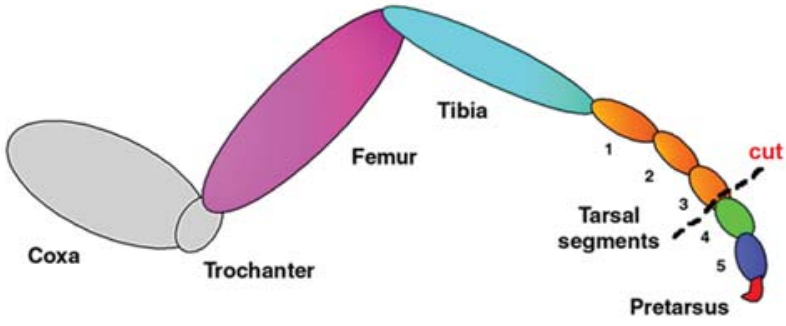

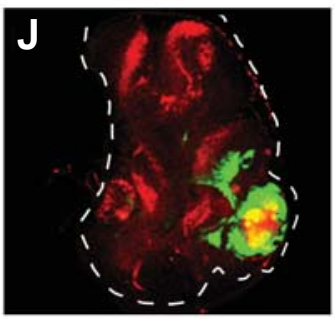
black dashed line indicates the site of the cut. (H) Disc amputated at $120 \mathrm{~h}$. AEL and then cultured showing formation of a cuticular scab (brown colour) over an unstained disc. (I) Regeneration of a proximal fragment cultured for 7 days showing duplicated distal structures, (ap green, al red). (J) Side view of a proximal leg disc fragment undergoing typical regeneration of the distal region after 7 days of culturing, showing a single focus of regenerating ap (green) and al expression (red, overlap in yellow). This typical regeneration is the basis for the results presented in this report (see text for details). Dashed line as in (D). disc. At this stage ap (green) is expressed, (staining as for D). (F) Triple staining in early pupal disc beginning to evert. ap (green) is expressed in the presumptive Ta4, overlapping the proximal part of the Bar domain (stained in blue; overlap appears pale blue, ar, but not the distal part of the Bar defines the presumptive Ta5 adjacent to the presumptive pretarsus (expressing al, red). Arrowheads point to adepithelial myoblasts expressing low levels of ap. Dashed line as in (D). (F') Green channel excluded, to show the entire Bar domain more clearly. (G) Diagrams showing amputation in a late third instar disc (left), and equivalent position in an adult leg (right), where 
Molecular data on insect limb or leg disc regeneration started with the observation that the $h h$ and $w g$ signalling cascades are active during the process (Brook etal., 1993; Gibson and Schubiger, 1999). More detailed information arose from new studies of terminal and intercalary leg regeneration in the cricket Gryllus bimaculatus (Mito et al., 2002; Nakamura et al., 2007, 2008; reviewed in Nakamura, 2008). As in Drosophilaleg development, the formation of a new PD axis is triggered at the site where healing of the cut surface brings together ventral wg-expressing cells and dorsal dpp-expressing cells in the newly established AP boundary (Mito et al., 2002). At this site, $W g+D p p$ activate DII. At later stages, $W g+D p p a n d$, likely, DI/activate EGFR signalling which is required for distal leg structures, the tarsus and the pretarsus (claws). Together with DI/, EGFR signalling activates $a /$ in the pretarsus (Nakamura et al., 2008).

In summary, the deployment of key signalling molecules in the early stages of leg disc development in Drosophila, and their redeployment in leg regeneration in other insects, are strikingly similar and consistent with the predictions of the BM (Meinhardt 1982, 1983) modified by Campbell and Tomlinson (CTBM; Campbell and Tomlinson, 1995). However, whether the spatial sequence of pretarsal and tarsal marker acquisition described during later development is similarly redeployed during regeneration had yet to be explored. During late development these markers are expressed from distal to proximal, apparently contravening the extrapolation of the 'distal transformation rule' observed previously in terminal regeneration. Thus, we have investigated the expression of eight marker genes, labelling tarsal and pretarsal segments, and their sequence of appearance during terminal regeneration in Drosophila leg discs. Moreover, the reversibility of cell fate specification, monitored by changes in gene expression, was also tested using lineage-tagged methods. We find that while the final patterns of gene expression along the PD axis of regenerated discs are similar to that of a fully developed imaginal disc, their temporal and spatial redeployment follows a proximal-to-distal sequence which is the opposite than during normal disc development. We also find

Fig. 2. Primary regenerative responses. (A) Late third-instar disc expressing apGal4-UASGFP (green) before amputation and (B) immediately after amputation, stained with phalloidin (red). In (B) ap expression is no longer visible due to distal amputation, and new expression has yet to be produced. Frontal view; dashed lines indicate the disc contour. (C) Expression of the JNK phosphatase puckered (puc, in green) in an amputated disc after 24h. of regeneration. Morphology of the disc is revealed with phalloidin (red). puc is extensively expressed over the disc but mainly follows the opposing wound edges, and the peripodial membrane (green staining outside the disc contour stained in red; see text and compare with control in D). Arrowhead indicates the centre of the wound. (C') Same as in (C) with green channel excluded. (D) Wild type puc (green, GFP) expression and anti-cleaved Caspase3 (b/ue) in a 110h. AEL third instar disc. puc expression is seen mainly in peripodial cells near the disc stalk (arrow), and cell death revealed by cleaved Cas3 occurs in a sparse pattern throughout the disc. Some overlap is observed at the presumptive femoral chordotonal organ (arrowhead). (D') Enlargement of the center of the disc in (D). White dashed line surrounds the tarsal knob, containing the presumptive pretarsus, Ta4 and Ta5. (E) Detail of a regenerating distal tip showing cell death 4 days after amputation, revealed with anti-cleaved Caspase 3 (Casp3, blue) with ap (green) and $\mathrm{C} 15$ (red) expression. $\mathrm{C} 15$ is engulfed by ap expression. Some dying cells in the regenerate are observed (arrow), but not at higher levels than unoperated controls (see $\left.D^{\prime}\right)$. (E') Same as in (D), green channel only. that proximal cells can readily adopt more distal identities and viceversa. The implications of similarities and differences between disc development and disc regeneration are discussed.

\section{Results}

Leg discs from 100-110 h.AEL larvae were dissected and their presumptive distal ends beyond tarsus three (Ta3) amputated (Fig. $1 \mathrm{G})$. Lack of ap-GFPexpression in cut discs was used to determine proper excision (Fig. 2 A,B) as apis expressed in tarsus four
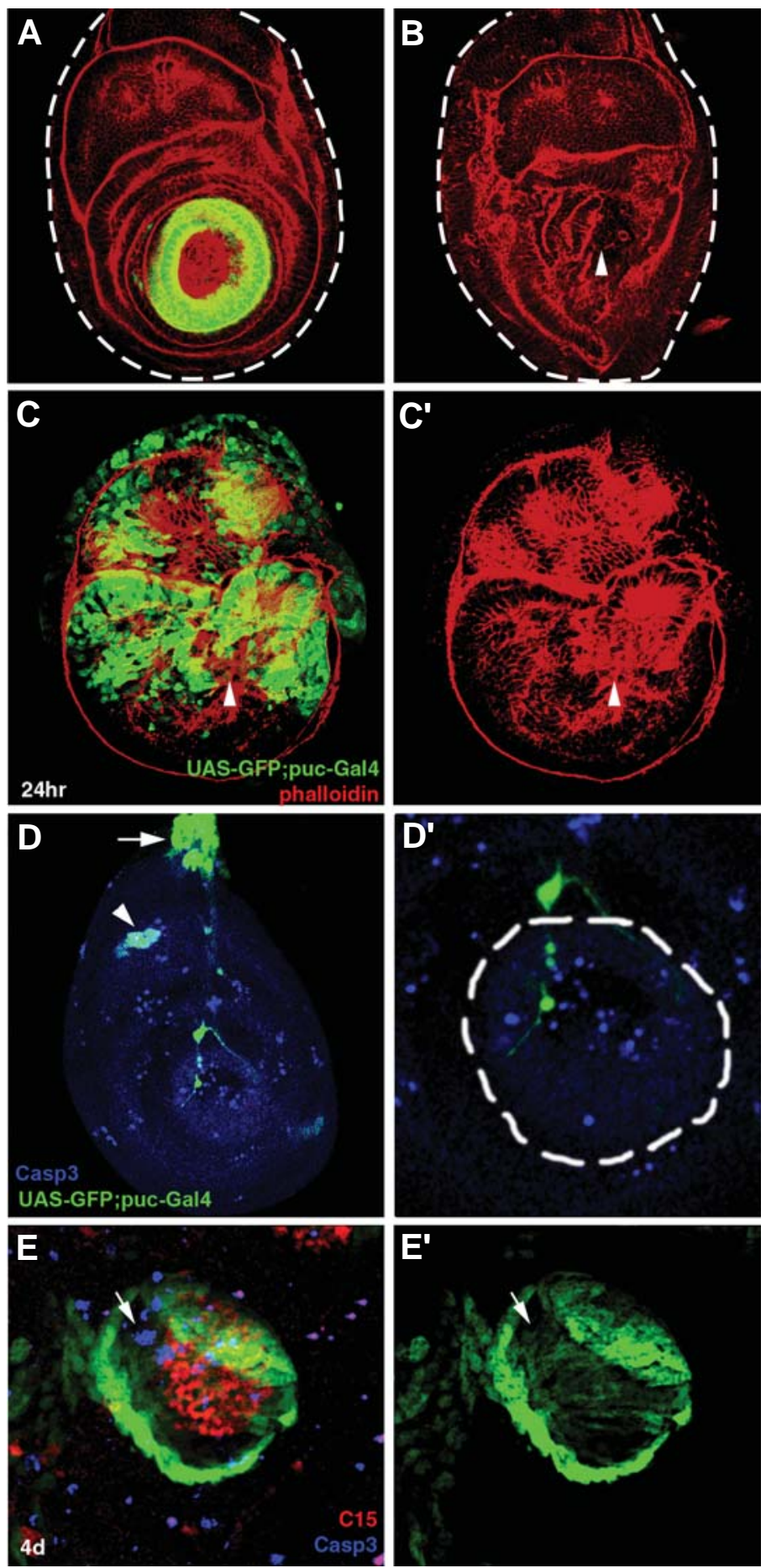
(Ta4). Pattern regeneration was studied by monitoring gene expression patterns removed or affected by the amputation: baband rnin proximal tarsal segments (Ta1-Ta4), ap in Ta4; Bin Ta5, and the pretarsal markers $C 15, a$, dlim 1 and the EGFR ligand $v n$ (Campbell, 2005; Kojima et al., 2005; Pueyo and Couso, 2004; Pueyo et al., 2000; Tsuji et al., 2000). Two types of control were used: normal developing discs, and unoperated discs transplanted and cultured as in the regeneration experiments. As with previous studies of disc regeneration, some variability was observed. In particular, discs amputated too late (and presumably exposed to the prepupal ecdysone pulse at 110 h.AEL) do not regenerate, but instead form a cuticular scab around the wound (Fig. 1H). Discs amputated between 100-110 h.AEL occasionally give rise to distal duplications (Fig. 1I) but usually regenerate a single distal end (Fig. $1 \mathrm{~J})$. Here we present only results that we observe to be reproducible.

\section{Primary regenerative responses}

24 hours after cutting, the circular wound generated by the amputation appeared completely healed. Wound healing in leg imaginal discs seems to occur, as in wing discs (Bosch etal., 2005), by zipping up the wound edges left by the cut giving rise to fragments with round morphologies. Closure of wound edges proceeds through formation and contraction of actin cables (Fig. 2 $\mathrm{B}, \mathrm{C}$ stained red with phalloidin). In addition, the JNK phosphatase, puckered (puc), which in regenerating wing discs highlights the regenerating cells close to the wound (Bosch et al., 2005; Mattila et al., 2005), is also induced in several rows of cells at the wound edges (Fig. 2C, marked with GFP). However, the larger wound area and its highly convoluted edges after distal removal in leg discs (as compared to wing disc fragmentation), together with the additional expression of puc in peripodial and other cells in leg discs (Fig. 2 C,D), makes puca less useful marker than in wing disc regeneration. Finally, some sparse cell death is observed both in controls (Fig. 2D) and in amputated discs (Fig. 2E), but it is not enhanced or prevalent in the regenerating tissue.

\section{Origin and proliferation of cells in leg disc regenerates}

Mitotic activity was checked and compared to early reports in regenerating legs, (Bryant and Fraser, 1988; Karpen and Schubiger,
Fig. 3. Origin and proliferation of cells in leg disc regenerates. (A) $\mathrm{Mi}$ totic patterns revealed by anti-H3P (red) in a wild-type leg disc (frontal view) with $\mathrm{rnGal} 4-\mathrm{U} A S G F P$ expression in green. (B) Disc immediately after amputation. $\mathrm{rn}$ expression remains at the wound edge (arrowhead) and mitosis remains unaffected. (C) Side view of regenerating disc. After 2 days of culture mitoses (red) are concentrated near the wound area (arrowhead) in the absence of ap epidermal expression. The green stained cells (arrow) are myoblasts inside the disc lumen, that express low levels of ap independently of epidermal development (see methods and compare with Fig. 1F). Dashed white line indicates disc contour. (D) After 4 days mitoses are more generally distributed throughout the disc. Regenerated ap epidermal expression is observed (arrowhead, green). Thick dash line as in $C$, thin dashed lines indicate the presumptive distal tip, magnified in the inset at the upper right hand corner. (E) Side view of a control leg disc (distal to the right) before cutting for the cell lineage tracing experiment. White dashed lines indicate the future plane of amputation. The tarsal ring of ap-GFP expression is in green. A substantial number of myoblast cells also express lacZ (asterisk). (E') Same as for (E) with green channel excluded. (F) Disc at 7 days of regeneration showing expression of ap and C15. Cells
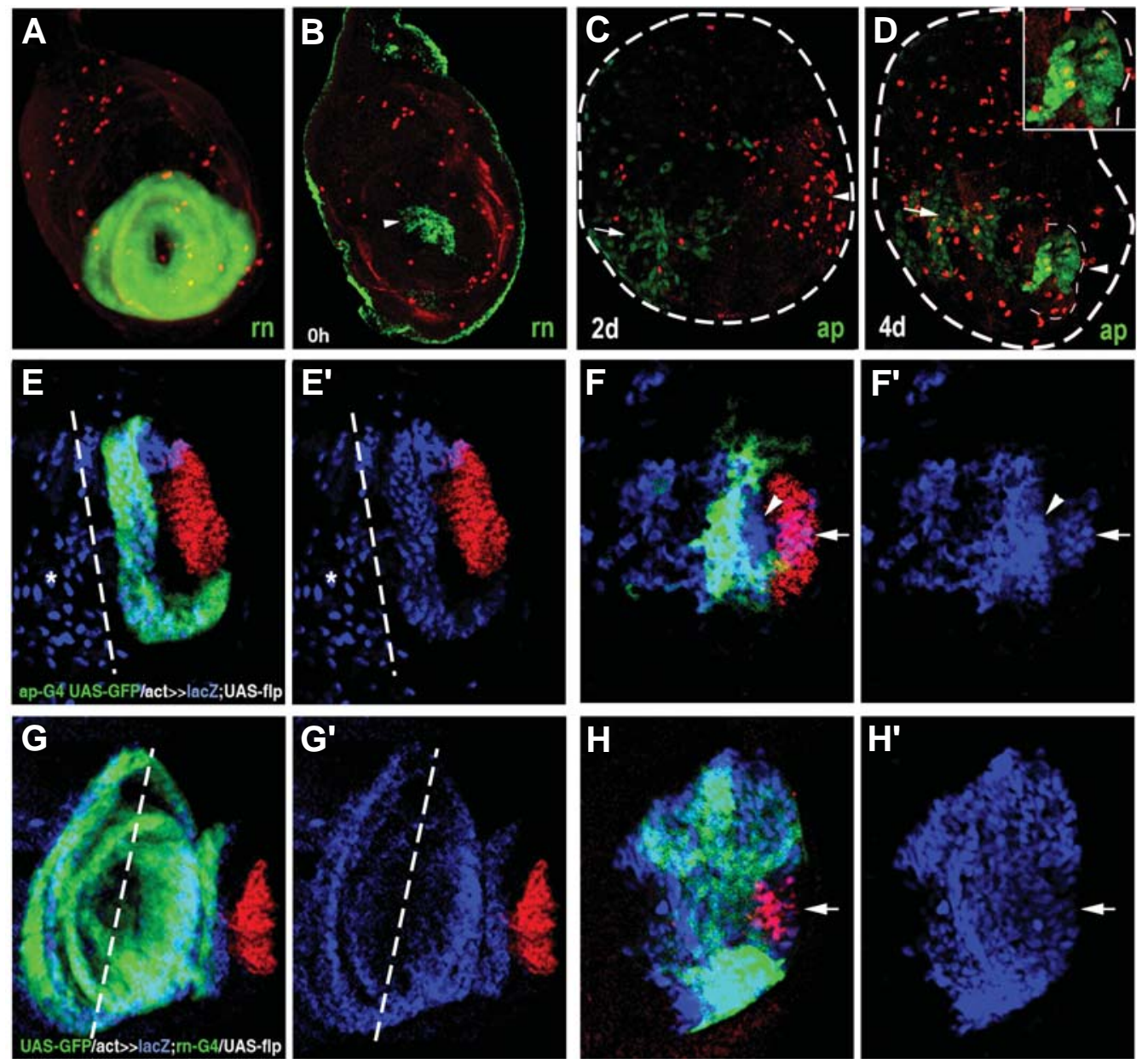

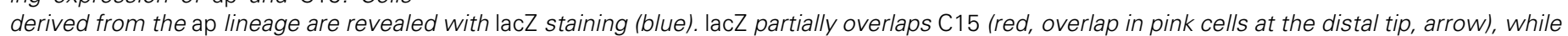

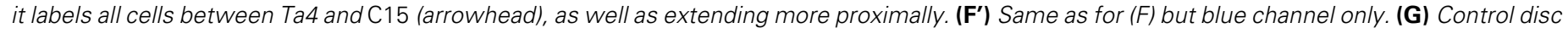

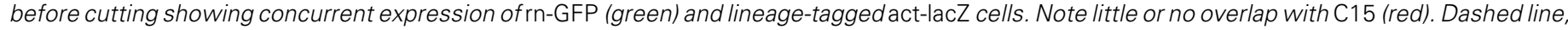

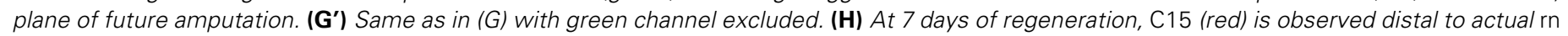
expression (green). lacZ expression is now present in all C15-expressing cells (arrow; overlap in pink). (H') Same as in (H), blue channel only. 
1981; Kiehle and Schubiger, 1985), and to recent data on regenerating wing imaginal discs (Bosch et al., 2008). While uncut and freshly cut discs show moderate and sparse mitotic activity (Fig. 3 $A, B), 2$-day regenerating discs show a clear increase in mitotic activity (Fig. 3C) in areas at and close to the wound. At 4 days of regeneration, mitotic activity, while higher than in unoperated discs, has a more scattered distribution. Interestingly, mitotic cells do occur in the regenerating distal domain (inset in Fig. 3D).

To ascertain the origin of cells in the regenerate we used a cell lineage-tracing method (Weigmann and Cohen, 1999; Bosch et al., 2008) in discs carrying apGal4 UAS-GFP/act-FRT-stop-FRTlacZ; UAS-Flp. In such discs, Flprecombinase, expressed in the ap domain, excises the flip-out 'stop' cassette from the inactive reporter construct to generate an active act>lac $Z$ transgene. Thus, constitutive lac $Z$ expression is clonally inherited in all the progeny of ap-Gal4-expressing cells in which the excision takes place. Meanwhile, GFP expression driven by ap-Gal4 indicates the cells in which the ap gene is actually active. In unoperated control discs, lacZexpression and GFP expression co-localize in the ring of ap-expressing cells (Fig. 3E). As expected, apand the pretarsal marker $C 15$ show completely non-overlapping expression. After cutting, neither lacZ nor GFP expression is detected (not shown). At 7 days of regeneration, expression of lacZoccurs in most of the regenerate including the distal-most regions, while GFP is restricted to a more proximal area forming the actual ring of regenerated ap expression (Fig. 3F). Interestingly, expression of lacZand expression of the distal (pretarsal) marker C15overlap extensively in the distal cells of the regenerate (see cells labelled in pink in Fig. 3F) while lacZlabels all cells between $C 15$ and Ta4 (where ap-driven GFP is expressed), (Fig. 3F'). This suggests that proximal-distal fates do not segregate from the very beginning of regeneration, but rather do so later on, as regeneration proceeds.

The origin of cells in the regenerate was also studied by lineage-tagging the expression of $r n G a / 4$. rnexpression overlaps ap but also extends more proximally (see Figs. 1 B,C and 3A); hence, cuts performed proximal to Ta4 fall within the rndomain, so that wound edge cells are $r n$ positive (Fig. 3B). In unoperated control discs, lacZexpression matches very closely the actual $r n$ expression (labelled with GFP) and no or little overlap with $C 15$ is detected (Fig. 3G). After 7 days of regeneration, lacZexpression occurs all over the regenerate while GFP expression (green label) does not occur in the most distal regions (Fig. 3H). Importantly, the extensive overlap between $C 15$-expressing cells and lacZ cells in 7 day regenerates indicates that all $C 15$-expressing cells derive from lacZexpressing cells (Fig. $3 \mathrm{H}, \mathrm{H}^{\prime}$ ). In addition, the non overlap between $C 15$ and $r n G a l 4$-driven GFP cells indicates that at 7 days of regeneration rnexpression has already ceased in the pretarsal cells of the regenerate.

Altogether, lineage-tagging with $a p$ and $r n$ strongly indicates
Fig. 4. Pattern formation during leg imaginal disc regeneration. In all cases apGal4 UAS-GFP is shown in green, and stainings in the red and blue channels are written on the left of the panels. Thick dashed lines indicate disc contours, and thin dashed lines indicate the presumptive distal leg proper. (A) Control intact disc after 7 days of culture showing the patterns of ap (green) and C15 (red). (B) Operated disc after 4 days of regeneration. New ap (green) and $\mathrm{C} 15$ (red) expression overlaps in some cells (yellow spots; arrows in $B^{\prime}$, $\left.B^{\prime \prime}\right)$. (B') Magnification of white box in (B). (B'") Same as (B') with red channel excluded. (C) After 7 days of regeneration, ap (green) and $\mathrm{C} 15$ (red) are expressed in different cells but very close to each other. (D) Control intact disc after 7 days of culture showing expression of ap (green), B (blue), and al (red). (D') Enlargement of the white box in (D). (D") Same as (D') with blue channel excluded. (E) Operated disc after 4 days of regeneration stained for ap (green) and al (red). ap is expressed at the regenerating tip (white box) while al is not, being only present in its proximal domain (arrow). (E') Enlargement of the white box in (E). (E") Same as $\left(E^{\prime}\right)$ with green channel excluded. (F) Enlargement of 7-day regenerating leg tip showing expression of ap (green), B (blue) and al (red). Arrows point to overlapping ap and al expression (overlap in yellow). Arrowhead indicates overlap of $\mathrm{B}$ and al expression (overlap in pink). (F', $\left.\mathbf{F}^{\prime \prime}, \mathbf{F}^{\prime \prime \prime}\right)$ Same as in (F) with exclusion of green, red and blue channels respectively. (G) Enlargement of another leg disc after 7 days of regeneration. Here B and ap do not overlap with al. (G') Same as in (G) with blue channel excluded.
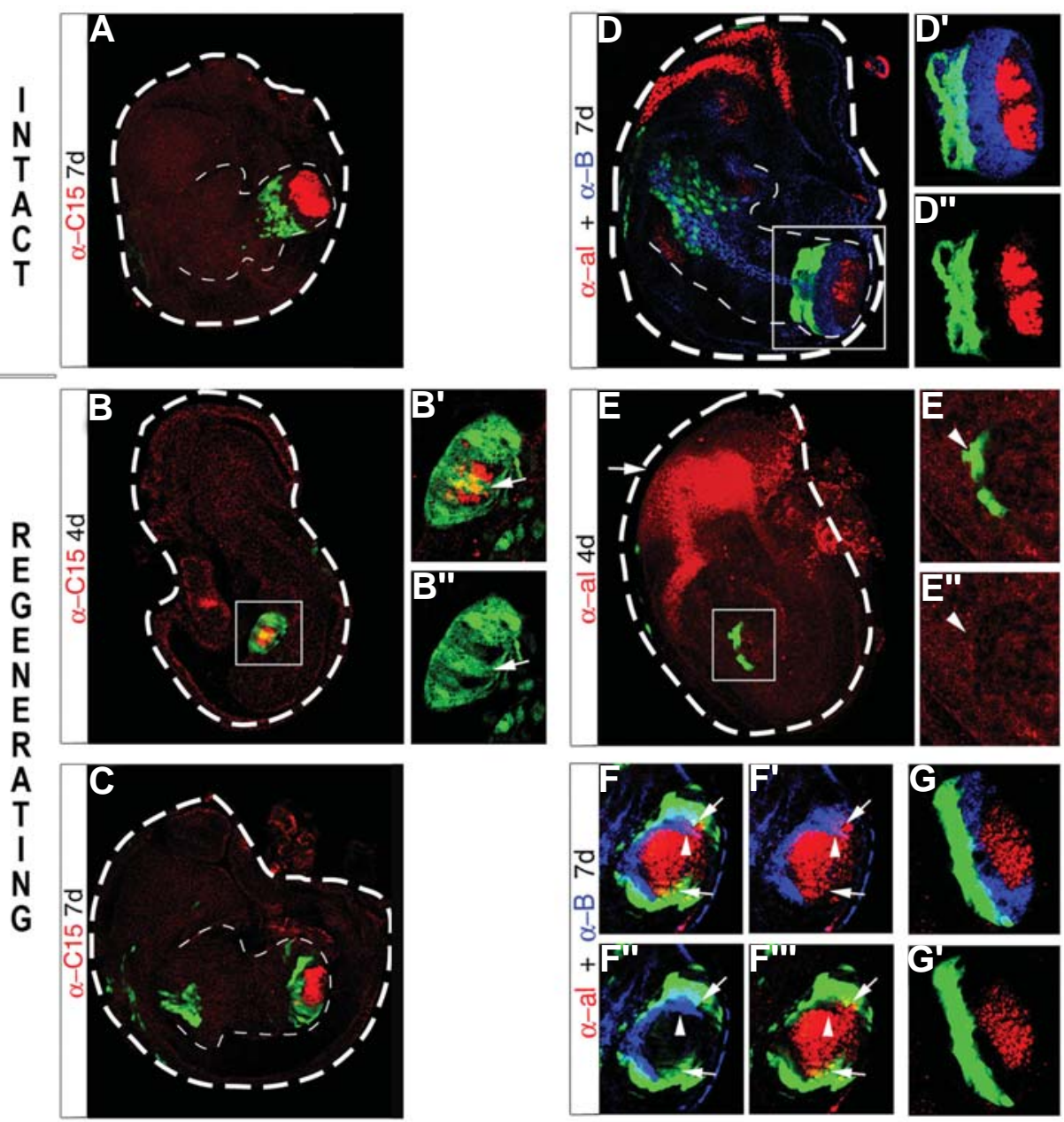

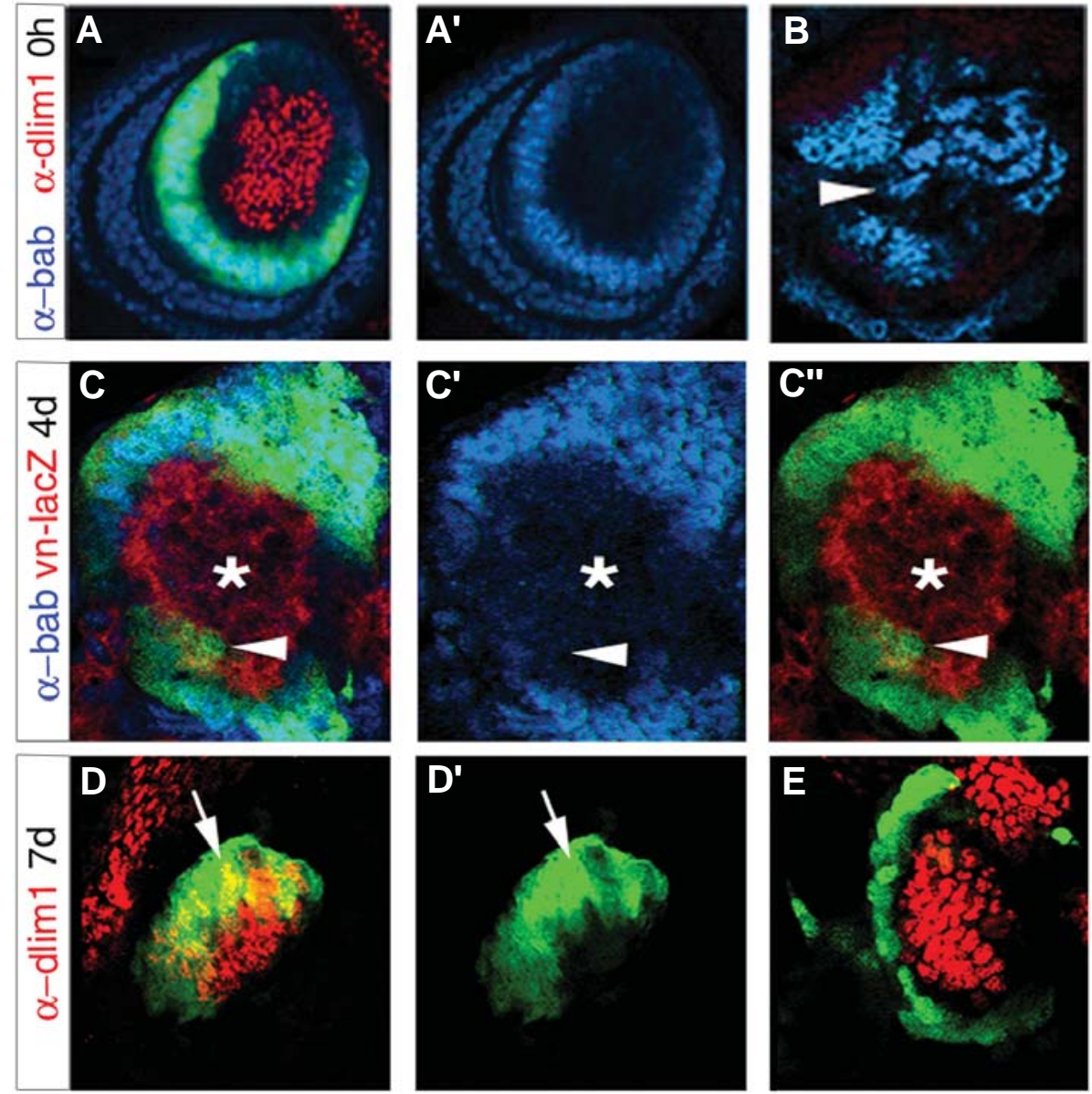

Fig. 5. Epidermal growth factor receptor (EGFR)-related gene expression during regeneration. In all cases, apGal4 UAS-GFP is showed in green, and staining in the red and blue channels is written on the left of the panels. (A) Control disc before cutting, showing ap (green) and bab (blue) co-localization in Ta4, and exclusion from the distal tip (dlim1, red). (A') Same as in (A), blue channel only. (B) Proximal fragment after cutting. Neither ap nor dlim1 expression remains. Arrowhead points out the centre of the wound. (C) Regenerating area after 4 days showing new ap expression (green) and decreased bab expression (blue) where vn expression has reappeared (red, and asterisk). Arrowhead, overlapping expression of ap and vn. (C') Same as in (C), blue channel only. (C') Same as in (C), blue channel excluded. (D) 7-day regenerating disc. dlim1 expression (red) overlaps more extensively with ap (green) than al (compare with Fig. 4F), or C15 (compare with Fig. 4B). (D') Same as (D) with red channel excluded. (E) Another regenerated tip, here with separated dim1 (red) and ap (green) domains.

that all cells in the regenerate originate from cells close to the wound. Hence regeneration occurs by the formation of a local blastema, (Odelberg, 2004). Moreover, the increase in mitotic activity near the wound and in the blastema further attest that growth of the regenerate basically results from local proliferation and not from cells proliferating and migrating from the rest of the disc. Finally, distal fates are regenerated from cells with a more proximal identity. We study this process in more detail in the next section using further molecular markers.

\section{Tracking pattern formation using molecular markers}

In control unoperated discs, the tarsal markers $a p$ (Ta4) and $B$ (Ta5) and the pretarsal markers $C 15$, dlim 1 and a/are expressed in a proximal-distal sequence of partially overlapping (i.e. $a p+B$ ) and non-overlapping domains (Fig. 4 A,D) as in wild-type discs

\section{Discussion}

(Fig. $1 \mathrm{D}-\mathrm{F}$ ). To infer how this pattern is restored during imaginal leg disc regeneration, we tracked these and other markers (see below) within regenerates. Expression of the pretarsal marker $C 15$ is observed at 4 days, though at variance with normal development it is found engulfed, and partially overlapped, by ap expressing cells (Fig. 4B). At 7 days of regeneration $a p$ and $C 15$ domains no longer overlap (Fig. 4C) though they are closer to each other than in unoperated discs (Fig. 4 $A, D)$. In contrast, a/ protein (Al), was not found in 4 day regenerates despite ap being already present (Fig. 4E); a situation unlike normally developing discs. At 7 days of regeneration, non-overlapping $a /$ and $a p$ domains, closer to each other than in unoperated discs, are clearly detected flanking a $B$-only domain between them (Fig. 4G). In some discs, however, ap and al do still overlap (arrows in Fig. 4F).

To further investigate the process of pattern formation during regeneration, three other markers were studied: $b a b$, a tarsal gene (Godt et al., 1993); dlim1, a pretarsal marker (Lilly et al., 1999; Pueyo et al., 2000; Tsuji et al., 2000), and vn, the EGFR pathway ligand expressed in the pretarsus (Schnepp et al., 1996; Campbell, 2002; Galindo et al., 2002). bab expression is similar to $r n$, and they both overlap each other and extend more proximally than $a p$ (Fig. 5A). As expected, bab is expressed in all cells around the wound after amputation (Fig. 5B), and in early blastema cells (not shown). At 4 days of regeneration, discs show decreased bab levels where vn expression reappears (Fig. 5C), although some overlaps are still visible. As occurs during normal development, this decrease suggests that $E G F R$ is repressing tarsal genes (Campbell, 2002; Galindo etal., 2002). In turn dlim1, which is an EGFR-dependent, late pretarsal marker (Galindo et al. 2005), was found to overlap with ap in scattered cells of some discs at 7 days of regeneration (Fig. 5D) whereas in other discs of this age their expression domains do not overlap (Fig. $5 \mathrm{E})$.

As seen in normal development (see Fig. 5A), control unoperated discs after 7 days in culture show non-overlapping expression patterns for these three markers (not shown). Therefore, and as seen above for $a p, C 15, a /$ and $B$, the transient overlapping patterns seen in regenerating discs after 7 days in culture cannot be considered artefacts of culture conditions.

We have used lineage-tagging methods and the expression of eight tarsal and pretarsal marker genes to a) track the origin of blastema cells, b) explore the reversibility of cell fate specification, 
and c) set out the order of appearance of these markers during leg imaginal disc regeneration and compare it to the sequence during normal development. The final aim was to test whether pattern formation during development and during regeneration achieve identical final products by similar or different mechanisms.

\section{The origin of blastema cells and the reversibility of cell fates}

The vast literature on thymidine and BrdU experiments showing cell proliferation at and close to the wound (e.g. Bryant and Fraser, 1988; Anderson and French, 1985; Kiehle and Schubiger, 1985; Truby, 1985), together with cell lineage tracking using wound cell markers, (e.g. the gene coding for the phosphatase of the JNK pathway puckered (puc), Bosch et al., 2008), clearly indicates that cells close to the wound are the main source of blastema cells in regeneration of Drosophila imaginal discs. Indeed, the lineage tagging experiments here reported using the markers rnand ap (Fig. $3 \mathrm{C}-\mathrm{H}$ ) have shown that cells derived from $r n$-and ap-driven lac Ztagged cells fill the entire blastema, while $r n$ and $a p$ actual gene expression appears restricted to more proximal regions. Altogether, this definitely proves earlier suggestions (Abbott et al., 1981) that systemic cells (wandering stem cells) or cells from areas far from the wound make no contribution to the blastema.

In this report we have also examined the ability of cells to change their developmental fate in response to amputation. Using the same lineage method, we find that cells expressing tarsal markers such as $b a b, r n$ and $a p$, lose their expression and activate the distalmost pretarsal markers as well as other markers of distal tarsal segments (e.g. Barin Ta5). This results in transitory, yet non-wild-type, overlaps of proximal and distal markers,

Fig. 6. Diagrammatic models of regulatory relationships of proximal-distal (PD) patterning genes in distal leg imaginal discs during development (A-D) and regeneration (E-H). Genetic interactions are drawn above and below each model respectively. (A) At second instar (LII) the distal leg is composed of antagonistic dac and DII domains established by wg and dpp (Lecuit and Cohen, 1997, Abu-Shaar and Mann, 1998). (B) At early third instar, EGFR signalling is activated, and together with DII induces B, C15 and al in the centre of the disc (Campbell 2002; Galindo et al., 2002, 2005). Antagonistic transcriptional repression between the pretarsal genes (C15, al and later dlim1) and Bar refines these domains (Campbell 2005; Kojima et al., 2000; 2005; Pueyo et al., 2000; Pueyo and Couso, 2004; Tsuji et al., 2000). (C) Later on, the tarsal genes ( $\mathrm{rn}$ and $\mathrm{bab}$ ) are activated by DII while inhibited distally by EGFR and proximally by dac (Galindo et al., 2002). (D) At mid-third instar ap is activated in Ta4 by the combination of Bar (Kojima et al., 2000, 2005; Pueyo et al., 2000; Pueyo and Couso, 2004), and tal (Pueyo and Couso 2008), while inhibited by the pretarsal genes indirectly via Notch signalling (De Celis Ibeas and Bray, 2003; Campbell, 2005; Kojima et al., 2005). (E) After cutting, the distalmost (wound) cells are tarsal cells, expressing $\mathrm{rn}$ and bab, and also DII, wg and dpp (not indicated). (F) At 3-4 days, vn expression reappears by the action of overlapping $\mathrm{wg}, \mathrm{dpp}$ and DII (Galindo et al., 2002; Mito et al., 2002; Nakamura et al., 2007, 2008; this work) and activates B via EGFR signalling. Since the regenerating cells have already been exposed to the tal signal (Pueyo et al., 2008), they respond immediately to $\mathrm{B}$ expression by activating ap (this work). (G) At later stages, higher EGFR levels activate the pretarsal genes (al, C15, dlim1) close to, or overlapping with, Bar and ap (Campbell, 2002; Galindo et al., 2002, 2005; this work). (H) Finally, pretarsal genes repress B and ap directly (Pueyo and Couso 2004; this work) and fill the centre of the disc, achieving the normal pattern. such as those between apand the distal pretarsus markers dlim1, a/and C15, and that of baband vn(Figs. 4 and 5). This indicates that proximal cells near the wound, or their descendants, lose their proximal fates and are induced to turn on the distal markers to provide the population of cells that contribute to the pretarsus and the tarsal segments distal to the amputation plane. Although some of these results could be due to perdurance of apGa/4- or rnGal4-driven GFP and not to proper gene expression, lineagetracking experiments clearly show that most of the $C 15$ cells, and the cells between $C 15$ and Ta4, descend from cells that had expressed apand rnat some point before or during regeneration. Interestingly, at 4 days and even at 7 days of regeneration some distal and proximal markers still show small areas of overlap.

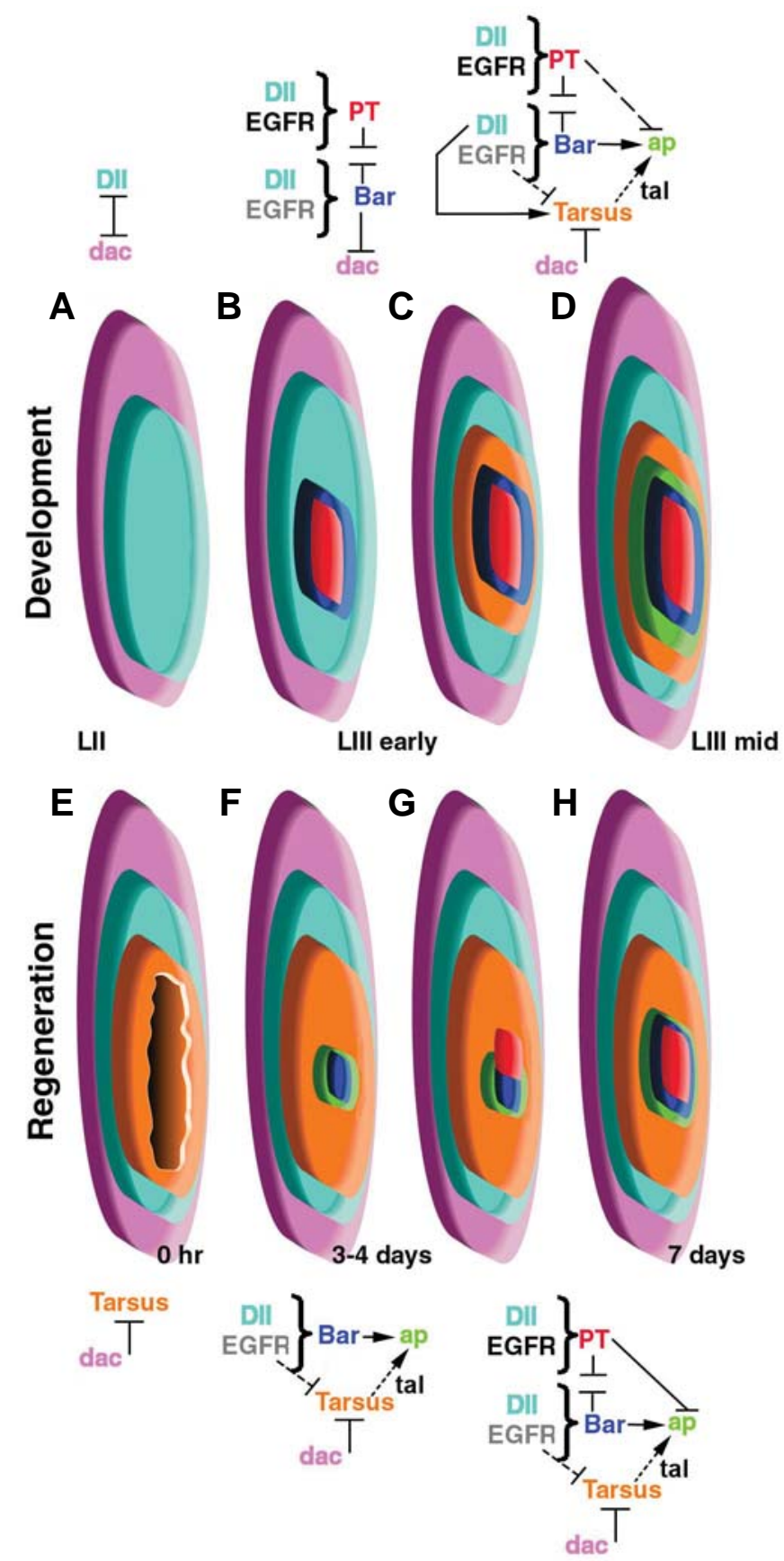


Thus ap/vnand ap/C15may overlap at 4 days (but not at 7 days), while ap/al, B/aland ap/dlim 1 still overlap in some discs at 7 days (Figs. 4 and 5). Importantly, in fully regenerated leg discs, tarsal and pretarsal (PT) domains never overlap. Furthermore, our results also indicate that the expression of distal markers is acquired in a proximal to distal sequence from ap-to- $C 15$ in contrast to the wild-type distal-to-proximal sequence that goes from C15to ap (see Fig. 6), (Campbell, 2005; Kojima et al., 2000; 2005; Pueyo and Couso, 2004; Pueyo et al., 2000; Tsuji et al., 2000).

Overall our results show that during terminal regeneration in Drosophilaleg imaginal discs, fates are regenerated in a proximal to distal sequence, and proximal cells switch their pattern of gene expression as the disc grows, to acquire distal fates. This fits an extrapolation of the 'distal transformation rule' proposed using morphological markers (French et al., 1976; Schubiger and Schubiger, 1978; Strub, 1979). It should be bourn in mind, however, that in several arthropods the reverse holds true during intercalary regeneration (Bohn, 1976; Truby, 1985; Mito et al., 2002), and during normal distal development. Thus 'distal transformation' might arise from a specific requirement to develop distal fates in the presence of pre-existing proximal ones, rather than as a result of hard-wired regeneration rules (see below).

\section{Regeneration follows specific developmental timings and emergent gene interactions}

Fig. 6 shows a comparison of cell signalling activity and PD patterning gene expression in the Drosophila leg imaginal disc during development (Fig. 6 A-D) and during regeneration (Fig. 6 $\mathrm{E}-\mathrm{H})$. In normal leg development, $B$ and the pretarsal markers al and $C 15$ are activated by a gradient of EGFRactivity in the centre of the D//domain at early third larval instar (72-80 h.AEL), (Fig. 6 A-B) (Campbell, 2002; Galindo et al., 2002). Subsequently, more proximal fates (tarsus 1-4) develop, through the activation of bab and $r n$ around $B$ by EGFR (Fig. $6 \mathrm{C}$ ), and by the transient activation of the tarsal-less (tal) gene by a $B$-dependent signal (Pueyo and Couso, 2008) from 80-96 h.AEL. The last PD gene to be expressed is ap, in the most proximal $B$ expressing cells (Ta4) by a $B$ and ta-dependent activation at 90-96 h.AEL (mid-third instar) (Pueyo and Couso, 2008). Expression of ap never overlaps with al, dlim1or C15(Fig. 6D), (Campbell, 2005; Kojima etal., 2005; Pueyo et al., 2000). If gene expression is considered an accurate indication of territorial determination, then PD pattern during development occurs by first setting the distalmost end (pretarsus and Ta5), followed by intercalation of proximal tarsal elements (Ta1-3) and finally by a distal one (Ta4). This contravenes the impression that limbs develop from proximal to distal, and the 'distal transformation' rule during regeneration, but upholds the rules of the boundary models with a distal organiser (Meinhart, 1983; Campbell and Tomlinson, 1995).

During terminal leg imaginal disc regeneration (Fig. $6 \mathrm{E}-\mathrm{H}$ ), the relationships between the markers observed here are as follows. After amputation, the baband rndomains (Ta1-3) are the most distal fates (Fig. 6E). After 3-4 days of regeneration, activation of $v n$ (presumably by $w g$ and $d p p$, which are active in regenerating discs, Brook et al., 1993; Gibson and Schubiger, 1999; Mito et al., 2002; unp. obs.), represses bab and seems to activate joint expression of $B$ and ap (Fig. 6F). Later on, EGFR activity is also required to activate the pretarsal genes al, dlim 1 and $C 15$ (Fig. 6G)

The expression of apbefore the pretarsal genes suggests that a signal already present in the tarsal cells of the proximal disc fragment is permitting ap expression in $B$ cells. During normal development this cell signal is triggered by taland acts between 84 and 96 h.AEL (Galindo et al., 2007; Pueyo and Couso, 2008). In regenerating discs a requirement for the ta/signal is suggested, as in normal development (Kojima et al., 2000; Pueyo and Couso, 2008 ), by the inability of ectopic $B$ to raise apexpression outside the regenerated tarsal region. In normal development, the requirement for ta/signalling delays apactivation; this delay allows segregation of pretarsal ( $C 15 /$ alldim 1 ) and $B$ territories, and hence, precludes overlaps of ap with pretarsal genes. However, during distal regeneration in 100-110 h.AEL discs, the blastemal tarsal cells have already been exposed to the normal ta/signal at 80-96 h.AEL, and are therefore competent to initiate ap expression as soon as $B$ expression reappears. This generates the overlaps observed between $a p$ and pretarsal genes. To resolve them, the pretarsal genes seem to repress $B$ directly, and might repress ap indirectly at a distance (through Notch signalling) as occurs during normal development (Campbell, 2005; Kojima et al., 2005; Pueyo and Couso, 2004). However ectopic expression experiments have revealed that the pretarsal genes (which encode transcription factors) also have the ability to repress the ap gene directly (Pueyo and Couso, 2004; Kojima et al., 2005), even though their expression patterns usually never overlap during normal development. This ability is also revealed during regeneration, and in this way, back-up, emergent properties of the normal developmental programme are exploited to resolve the new situation created during regeneration.

The regenerative programme is not perfect. The presence of Ap protein leads to the formation of $A p / B$ dimers (Pueyo and Couso, 2004) that lower the availability of $B / B$ homodimers to counteract repression by $\mathrm{C} 15 / \mathrm{Al} / \mathrm{Dlim} 1$ complexes and promote Ta5 determination (Kojima et al., 2005; Pueyo and Couso, 2004). Thus the co-localization of $A p$ and $B$ reduces the pool of B-only cells necessary for Ta5 determination. This effect reduces the size of Ta5 and compromises the orderly differentiation of coherent tarsus 4, 5 and pretarsus territories (Pueyo and Couso, 2004; Kojima et al., 2005). Accordingly, tarsus 4 and 5 are often fused after regenerative development in insects (Karpen and Schubiger, 1981; Tanaka et al., 1992; unp. obs.).

Taken together, our results show that regeneration takes advantage of the remarkable resilience of developmental programmes. They suggest that during leg regeneration cells find themselves in abnormal situations, requiring them to bridge together a developmental programme that generates fates from the distal tip with a regenerative growth driven from the proximal end. Our work generally upholds the 'distal transformation rule', but also reveals the adaptable way in which it is accomplished. Our observations do not fully favour an orderly 'Proximal to Distal' mode of regeneration. Lineage-tracing experiments show that the leg tip develops from more than just ap-expressing cells, and also gene expression markers reveal near-simultaneous activation of several PD markers. In our experiments PD gene expression patterns can reappear mixed-up, and proper PD fates only segregate after secondary gene interactions, in concert with expansion of the regenerate through cell proliferation. This interpretation could also apply to overlapping Hox expression in regenerating 
amphibian limbs (Gardiner et al., 1995; Carlson et al., 2001), corroborating again the similarity between both systems. The question arises as to why normal development proceeds in its particular way when regeneration offers an alternative. A likely answer might be that during regeneration a higher degree of variability in the patterns of gene expression is the rule, and often only an approximate optimal final situation is achieved. Hence the 'normal development' route appears as a more robust and reliable process that still benefits from the back-up properties that regeneration seems to exploit.

\section{Materials and Methods}

\section{Drosophila stocks and genetics}

Fly cultures were grown on standard medium at $25^{\circ} \mathrm{C}$. Reporter alleles used were: $v n^{R f} / a c Z$ (Galindo et al., 2002); apGal4 (Calleja et al., 1996); rnGal4 and rnlacZ (St Pierre et al., 2002). Genetic markers and chromosome balancers are described in FlyBase (http://flybase.bio.indiana.edu).

\section{Surgery and in vivo disc culture}

Male foreleg imaginal discs were removed from wandering larvae (100-110 hours after egg laying; h.AEL, identified by behaviour and morphology) in Schneider's insect medium (Sigma), and cut with tungsten needles across the PD axis proximal to apGal4 UAS-GFPexpression in tarsus 4 (Ta4). Dissection was performed under a fluorescence microscope. apis also detected in adepithelial myoblasts but this expression is weaker and easily distinguished (see Fig. 1F). Following established procedures (Schubiger, 1978), experimental (ap-fragments) and unoperated (uncut) discs were implanted into the abdomens of recently eclosed Canton $\mathrm{S}$ females and kept at $25^{\circ} \mathrm{C}$. Following a culture period of 2 , 4 , or 7 days, implanted discs were removed from the hosts and analysed.

\section{Cell lineage experiments}

To explore the origin of cells in regenerates of proximal leg fragments we used a cell lineage-tracing method (Weigmann and Cohen, 1999; Bosch et al., 2008) in leg discs of two different genotypes. In the first, Act5c-FRT-stop-FRT-lacZ;UAS-flipase/TM6Bflies were crossed to ap Gal4 $U A S-G F P / C y O$ and leg discs from the larval offspring were cut proximally to the GFP ring in Ta4 as above. In the second, Act5c-FRT-stop-FRTlacZ;UAS-flipase/TM6Bline was crossed to UAS-GFP;rnGa/4/TM6Band leg discs from the larval offspring were also cut proximally to Ta4, using the tarsal folds as morphological markers. Proximal fragments were implanted into adult hosts and left to regenerate for 7 days before analysis. In these experiments, FLP recombinase is expressed either in the apdomain or in the rndomain, excises the flip-out 'stop' cassette, and generates an active actin>lacZtransgene which is clonally inherited in all progeny of ap-Gal4or $r n$-Gal4-expressing cells in which the excision took place. To detect lacZexpression, an anti- $\beta$-galactosidase antibody (1:1000, Cappel) was used and detected with a donkey anti-rabbit rhodaminelabelled secondary antibody (1:200, Jackson Immunoresearch). GFP activity driven by apor rnwas monitoredby GFP fluorescence. ap shows two distinct levels of expression (as in wings), low in adepithelial cells and high in epidermal cells.

\section{Immunohistochemistry}

Antibody staining was carried out as reported in Bishop et al. (1999). Primary antibodies were: anti- $\beta g a /$ (rabbit, 1:1000, Cappel), antiPhosphoHistone3 (rabbit, 1:1000, Upstate Biotechnology), anti-C15 (1:1000, Campbell, 2005), anti-Al (rat, 1:1000, Campbell et al., 1993), anti-B (rabbit, 1:20, Higashijima et al., 1992), anti-Dlim1 (guinea-pig, 1:1000, Lilly et al., 1999) and anti-Bab (rat, 1:800, Godt et al., 1993). Secondary antibodies conjugated with FITC or rhodamine were provided by Jackson Immunoresearch and used 1:200. Incubation was done at
Room Temperature for $2 \mathrm{~h}$. or overnight at $4^{\circ} \mathrm{C}$. Discs were analysed with a Zeiss LSM 510 confocal microscope. Images were processed with ImageJ (NIH Image; www.rsb.info.nih.gov/ij) and Photoshop 7.0 (Adobe Corporation).

\section{Acknowledgements}

We thank Iñaki Pueyo for unpublished results, and the rest of the Couso lab for discussions and help; and Rob Ray, Robert Whittle, F. Serras, and M. Corominas for comments and suggestions. This work was funded by a Wellcome Trust Senior Fellowship to JPC (GR057730MA), a Marie-Curie Training Site Grant (HPMT-CT-2001-00232), a predoctoral grant from the Universitat de Barcelona to $M B$, and by grants from Ministerio de Ciencia y Tecnologia, and Generalitat de Catalunya to JB.

\section{References}

ABBOTT, L.C., KARPEN, G.H. and SCHUBIGER, G. (1981). Compartmental restrictions and blastema formation during pattern regulation in Drosophila imaginal leg discs. Dev Bio/87: 64-75.

ABU-SHAAR, M. and MANN, R. S. (1998). Generation of multiple antagonistic domains along the proximodistal axis during Drosophila leg development. Development 125: 3821-3830

ANDERSON, H. and FRENCH, V. (1985). Cell division during intercalary regeneration in the cockroach leg. Embryol. exp. Morph. 90: 57-78.

BART, A. (1988). Proximodistal leg regeneration in Carausius morosus: growth intercalation and proximalization. Development 102: 71-84.

BASLER, K. and STRUHL, G. (1994). Compartment boundaries and the control of Drosophila limb pattern by hedgehog protein. Nature 368: 208-214.

BISHOP, S. A., KLEIN, T., MARTINEZ-ARIAS. A. and COUSO, J. P. (1999). Composite signalling from Serrate and Delta establishes leg segments in Drosophila through Notch. Development 126: 2993-3003.

$\mathrm{BOHN}, \mathrm{H}$. (1976). Regeneration of proximal tissues from a more distal amputation level in the insect leg (Blaberus craniffer, Blattaria). Dev Biol. 53: 285-293.

BOSCH, M., SERRAS, F., MARTIN-BLANCO, E. and BAGUÑÀ, J. (2005). JNK signalling pathway required for wound healing in regenerating Drosophilawing imaginal discs. Dev Bio/280: 73-86.

BOSCH, M., BAGUÑÀ, J. and SERRAS, F. (2008). Origin and proliferation of blastema cells during regeneration of Drosophila wing imaginal discs. Int. J. Dev. Bio/52: 1043-1050.

BROOK WJ, OSTAFICHUK LM, PIORECKY J, WILKINSON MD, HODGETTS DJ, RUSSELL MA (1993). Gene expression during imaginal disc regeneration detected using enhancer-sensitive P-elements. Development 117:1287-1297

BRYANT, S. V., FRENCH, V. and BRYANT, P. J. (1981). Distal regeneration and symmetry. Science 212: 993-1002.

BRYANT, P. J. and FRASER, S. E. (1988). Wound healing, cell communication, and DNA synthesis during imaginal disc regeneration in Drosophila. Dev Bio/127 197-208

CALLEJA, M., MORENO, E., PELAZ, S. and MORATA, G. (1996). Visualization of gene expression in living adult Drosophila. Science 274: 252-255

CAMPBELL, G., WEAVER, T. and TOMLINSON, A. (1993). Axis specification in the developing Drosophila appendage: the role of wingless, decapentaplegic, and the homeobox gene aristaless. Cel/74: 1113-1123.

CAMPBELL, G. and TOMLINSON, A. (1995). Initiation of the proximodistal axis in insect legs. Development 121: 619-28.

CAMPBELL, G. (2002). Distalization of the Drosophilaleg by graded EGF-receptor activity. Nature 418: 781-5.

CAMPBELL, G. (2005). Regulation of gene expression in the distal region of the Drosophila leg by the Hox11 homolog, C15. Dev Bio/278: 607-618.

CARLSON, M. R., KOMINE, Y., BRYANT, S. V. and GARDINER, D. M. (2001). Expression of Hoxb13 and Hoxc10 in developing and regenerating Axolotl limbs and tails. Dev Bio/229: 396-406.

COUSO, J. P., BATE, M. and MARTINEZ-ARIAS, A. (1993). A wingless-dependent polar coordinate system in Drosophila imaginal discs. Science 259: 484-489.

COUSO, J. P. and BISHOP, S. A. (1998). Proximo-distal development in the legs 
of Drosophila. Int J Dev Bio/42: 345-352.

DE CELIS IBEAS, J. M., and BRAY, S. J. (2003). Bowl is required downstream of Notch for elaboration of distal limb patterning. Development 130: 5943-5952.

DIAZ-BENJUMEA, F.J., COHEN, B. and COHEN, S.M. (1994). Cell interaction between compartments establishes the proxomal-distyal axis of Drosophila legs. Nature 372: 175-179.

FRENCH, V., BRYANT, P. J. and BRYANT, S. V. (1976). Pattern regulation in epimorphic fields. Science 193: 969-981.

GALINDO, M. I. and COUSO, J. P. (2000). Intercalation of cell fates during tarsal development in Drosophila. Bioessays 22: 777-780.

GALINDO, M. I., BISHOP, S. A., GREIG, S. and COUSO, J. P. (2002). Leg patterning driven by proximal-distal interactions and EGFR signaling. Science 297: 256-259.

GALINDO, M. I.,BISHOP, S. A. and COUSO, J.P. (2005). Dynamic EGFR-Ras signalling in Drosophila leg development. Dev Dyn 233: 1496-1508.

GALINDO, M. I., PUEYO, J. I., FOUIX, S., BISHOP, S. A., and COUSO, J. P. (2007). Peptides encoded by short ORFs control development and define a new eukaryotic gene family. Plos Biology 5: 1052-1062.

GARDINER, D. M., BLUMBERG, B., KOMINE, Y. and BRYANT, S. V. (1995). Regulation of HoxA expression in developing and regenerating axolotl limbs. Development 121: 1731-1741.

GIBSON, M.C and SCHUBIGER, G. (1999). Hedgehog is required for activation of engrailed during regeneration of fragmented Drosophila imaginal discs. Deve/opment 126:1591-1599.

GODT, D., COUDERC, J. L., CRAMTON, S. E. and LASKI, F. A. (1993). Pattern formation in the limbs of Drosophila: bric a brac is expressed in both a gradient and a wave-like pattern and is required for specification and proper segmentation of the tarsus. Development 119: 799-812.

KARPEN, G. H. and SCHUBIGER, G. (1981). Extensive regulatory capabilities of a Drosophila imaginal disk blastema. Nature 294: 744-747.

KIEHLE, C. P. and SCHUBIGER, G. (1985). Cell proliferation changes during pattern regulation in imaginal leg discs of Drosophila melanogaster. Dev Biol 109: 336-346.

KOJIMA, T., SATO, M. and SAIGO, K. (2000). Formation and specification of distal leg segments in Drosophila by dual Barhomeobox genes, BarH1 and BarH2. Development 127: 769-778.

KOJIMA, T. (2004). The mechanism of Drosophila leg development along the proximodistal axis. Dev Growth Differ 46: 115-129.

KOJIMA, T., TSUJI, T. and SAIGO, K. (2005). A concerted action of a paired-type homeobox gene, aristaless, and a homolog of Hox $11 / \mathrm{tl}$ x homeobox gene, clawless, is essential for the distal tip development of the Drosophila leg. Dev Bio/279: 434-445.

LECUIT, T. and COHEN, S.M. (1997). Proximal-distal axis formation in the Drosophila leg. Nature 388: 139-145.

LILLY, B., O'KEEFE, D. D., THOMAS, J. B. and BOTAS, J. (1999). The LIM homeodomain protein dlim1 defines a subclass of neurons within the embryonic ventral nerve cord of Drosophila. Mech Dev 88: 195-205.

MATTILA, J., OMELYANCHUK, L., KYTTALA, S., TURUNEN, $H$. and NOKKALA, S. (2005). Role of Jun N-terminal Kinase (JNK) signaling in the wound healing and regeneration of a Drosophila melanogasterwing imaginal disc. Int $J \mathrm{Dev}$ Bio/49: 391-399.

MEINHARDT, H. (1982). Models of biological pattern formation. Academic Press, London.
MEINHARDT, H. (1983). Cell determination boundaries as organizing regions for secondary embryonic fields. Dev Bio/96: 375-385.

MITO, T., INOUE, Y., KIMURA, S., MIYAWAKI, K., NIWA, N., SHINMYO, Y., OHUCHI, H. and NOJI, S. (2002). Involvement of hedgehog, wingless, and dpp in the initiation of proximodistal axis formation during the regeneration of insect legs, a verification of the modified boundary model. Mech Dev 114: 27-35.

NAKAMURA, T., MITO, T., TANAKA, Y., BANDO, T., OHUCHI, H. and NOJI, S (2007). Involvement of canonical Wnt/Wingless signalling in the determination of the positonal values within the leg segment of the cricket Gryllus bimaculatus. Develop Growth Differ 49: 79-88.

NAKAMURA, T., MITO, T., MIYAWAKI, K., OHUCHI, H. and NOJI, S. (2008). EGFR signalling is required for re-establishing the proximodistal axis during leg regeneration in the cricket Gryllus bimaculatus nymph. Dev Bio/319: 46-55.

NAKAMURA, T., MITO, T., BANDO, T., OHUCHI, H. and NOJI, S. (2008). Dissecting insect leg regeneration through RNA interference. Cell. Mol. Life Sci. 65: 6472.

ODELBERG, S. J. (2004). Unraveling the molecular basis for regenerative cellular plasticity. PLOS Biol 2: e232.

PUEYO, J. I., GALINDO, M. I., BISHOP, S. A. and COUSO, J. P. (2000). Proximaldistal leg development in Drosophila requires the apterous gene and the Lim1 homologue dlim1. Development 127: 5391-5402.

PUEYO, J. I. and COUSO, J. P. (2004). Chip-mediated partnerships of the homeodomain proteins Bar and Aristaless with the LIM-HOM proteins Apterous and Lim1 regulate distal leg development. Development 131: 3107-3120.

PUEYO, J. I. and COUSO, J.P. (2008). The 11-aminoacid long Tarsal-less peptides trigger a cell signal in Drosophila leg development. Dev. Biol. 324: 192-201.

SANCHEZ ALVARADO, A. (2000). Regeneration in the metazoans: why does it happen? BioEssays 22: 578-590.

SCHNEPP, B., GRUMBLING, G., DONALDSON, T., and SIMCOX, A. A. (1996). Vein is a novel component in the Drosophila epidermal growth factor receptor pathway with similarity to the neuregulins. Genes Dev 10:2302-2313

SCHUBIGER, G. and SCHUBIGER, M. (1978). Distal transformation in Drosophila leg imaginal disc fragments. Dev Biol. 67: 286-295.

ST PIERRE, S. E., GALINDO, M. I., COUSO, J. P. and THOR, S. (2002). Contro of Drosophila imaginal disc development by rotund and roughened eye: differentially expressed transcripts of the same gene encoding functionally distinct zinc finger proteins. Development 129: 1273-1281.

STRUB, S. (1979). Leg regeneration in insects. An experimental analysis in Drosophila and a new interpretation. Dev Bio/69: 31-45.

TANAKA, A., AKAHANE, H. and BAN, Y. (1992). The problem of the number of tarsomeres in the regenerated cockroach leg. J Exp Zoo/262: 61-70.

TRUBY, P.R. (1985). Separation of wound healing from regeneration in the cockroach leg. J. Embryol. Exp. Morph. 85: 177-190.

TSUJI, T., SATO, A., HIRATANI, I., TAIRA, M., SAIGO, K. and KOJIMA, T. (2000) Requirements of Lim1, a Drosophila LIM-homeobox gene, for normal leg and antennal development. Development 127: 4315-4323.

WEIGMANN, K. and COHEN, S. M. (1999). Lineage-tracing cells born in different domains along the PD axis of the developing Drosophilaleg. Development 126 : 3823-3830.

WU, J. AND COHEN, S. M. (1999). Proximodistal axis formation in the Drosophila leg: subdivision into proximal and distal domains by Homothorax and Distalless. Development 126: 109-117. 


\section{Further Related Reading, published previously in the Int. J. Dev. Biol.}

See our recent Special Issue Placenta edited by Joan S. Hunt and Kent L. Thornburg at: http://www.ijdb.ehu.es/web/contents.php?vol=54\&issue=2-3

Planarian regeneration: achievements and future directions after 20 years of research

Emili Saló, Josep F. Abril, Teresa Adell, Francesc Cebriá, Kay Eckelt, Enrique Fernández-Taboada, Mette Handberg-Thorsager, Marta Iglesias, M Dolores Molina and Gustavo Rodríguez-Esteban

Int. J. Dev. Biol. (2009) 53: 1317-1327

Regeneration and pattern formation - an interview with Susan Bryant

Michael K. Richardson and Cheng Ming Chuong

Int. J. Dev. Biol. (2009) 53: 827-833

Diffusible gradients are out - an interview with Lewis Wolpert

Michael K. Richardson

Int. J. Dev. Biol. (2009) 53: 659-662

Origin and proliferation of blastema cells during regeneration of Drosophila wing imaginal discs

Manel Bosch, Jaume Baguñà and Florenci Serras

Int. J. Dev. Biol. (2008) 52: 1043-1050

Role of Jun N-terminal Kinase (JNK) signaling in the wound healing and regeneration of a Drosophila melanogaster wing imaginal disc

Jaakko Mattila, Leonid Omelyanchuk, Satu Kyttälä, Heikki Turunen and Seppo Nokkala

Int. J. Dev. Biol. (2005) 49: 391-399

Dynamics of decapentaplegic expression during regeneration of the Drosophila melanogaster wing imaginal disc Jaakko Mattila, Leonid Omelyanchuk and Seppo Nokkala

Int. J. Dev. Biol. (2004) 48: 343-347
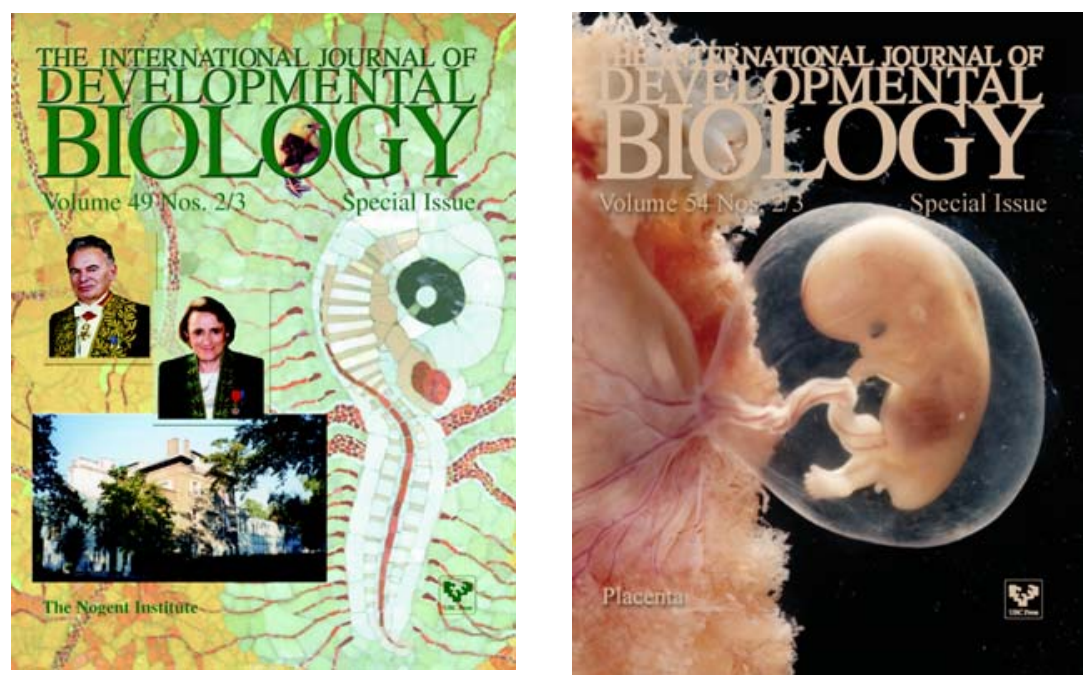

5 yr ISI Impact Factor $(2009)=3.253$
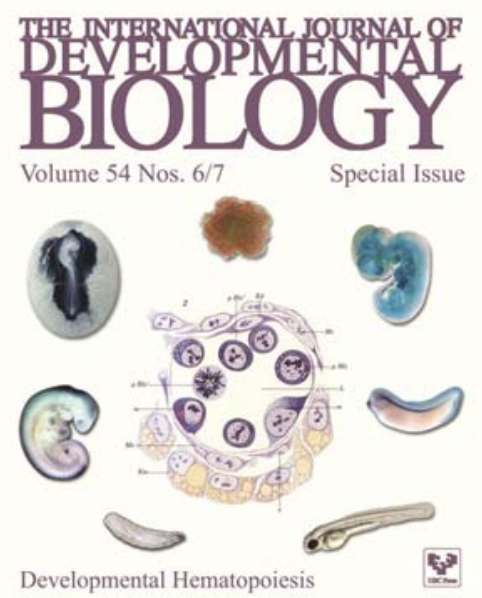